\title{
TIME-LAPSE MONITORING OF CELL MECHANICAL PROPERTIES
}

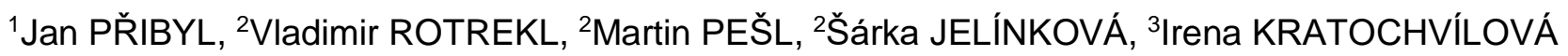 \\ ${ }^{1}$ CEITEC MU, CF Nanobiotechnology, Masaryk University, Brno, Czech Republic, EU, \\ jan.pribyl@ceitec.muni.cz \\ ${ }^{2}$ Department of Biology, Faculty of Medicine, Masaryk University, Brno, Czech Republic, EU, \\ vrotrekl@med.muni.cz \\ 3/nstitute of Physics, Academy of Sciences Czech Republic v.v.i, Prague, Czech Republic, EU, krat@fzu.cz
}

https://doi.org/10.37904/nanocon.2020.3740

\begin{abstract}
Atomic force microscopy (AFM) is a highly sensitive non-invasive surface method able to provide insight into cells' mechanical parameters. Membrane and sub-membrane development, as well as internal cellular properties, can be monitored. The stiffness of cells is a fundamental phenomenon that reflects changes in cell physiology. More importantly, changes in cell mechanical properties are also often found to be closely associated with various disease conditions. Cell mechanics are mainly dependent on cytoskeletal architecture. The development of cryopreserved cells' mechanical properties (stiffness) after thawing was studied using AFM. Cell stiffness was mapped and thus monitored in time and space under nearly physiological conditions (i.e., in culture medium and at elevated temperature). In AFM force spectroscopy mode, cells are indented at many sites, and their complete elastic responses are recorded, enabling them to reconstruct a stiffness map. We measured the frozen cell surface stiffness immediately after thawing; they, when the dynamics of development of the cell stiffness were monitored in time up to 24 hours. Moreover, the AFM spectroscopy was combined with fluorescence-based staining of the cytoskeleton, thus enabling to directly correlate cytoskeleton development with stiffness mapping.
\end{abstract}

Keywords: Atomic Force Microscopy, Mechanical Mapping, Cell stiffness, Cryopreservation

\section{INTRODUCTION}

Characterization of cell mechanical properties [1] help to understand many biological process, including cell cytoskeleton development [2] and remodeling [3], cell membrane structure, overall cell development and cell death [4]. Cell stiffness is regarded to the development of cytoskeleton assembly, myosin activity, nucleus state and processes $[5,6]$. Actin filaments structure is a basic indicator of cell development and adhesion properties. Many critical cellular properties, such tumor formation and development, are mirrored in the changes of mechanical properties [7]. Similarly, the cytoskeleton mechanical properties can be used for sensing of cell apoptosis, moreover, actin filaments organization was shown as relevant to breast cell phenotype [8].

There is a limited number of methods available for the investigation of the cell stiffness under physiological conditions, such as Brillouin microscopy [9], holographic microscopy [4], micropipette aspiration [10] or microwave propagation [11]. Atomic Force Microscopy (AFM) presents a method, which can be easily applied to the mechanical characterization of living cells adhered on solid surface under so called nearly physiological conditions. Moreover, high resolution mapping with the AFM probe is easily offered, maps of Young's modulus are reconstructed, when the force-distance curve measured within the network of the points are fitted with the appropriate mathematical model [6]. 
In this work, we studied stiffness of cryopreserved frozen/thawed cells and process of these cell mechanical properties/cytoskeleton regeneration after freezing/thawing. Ability of the AFM based nano-indentation technology for time lapsed monitoring of the cell mechanical properties, thus served as tool for mechano-related cellular processes. Overall description of the whole experimental concept can be inspected in the Figure 1.

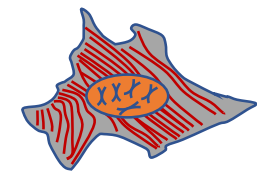

Fibroblast

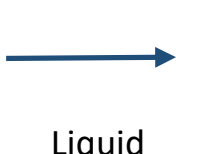

Liquid

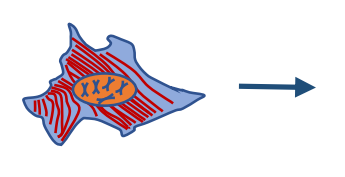

Frozen
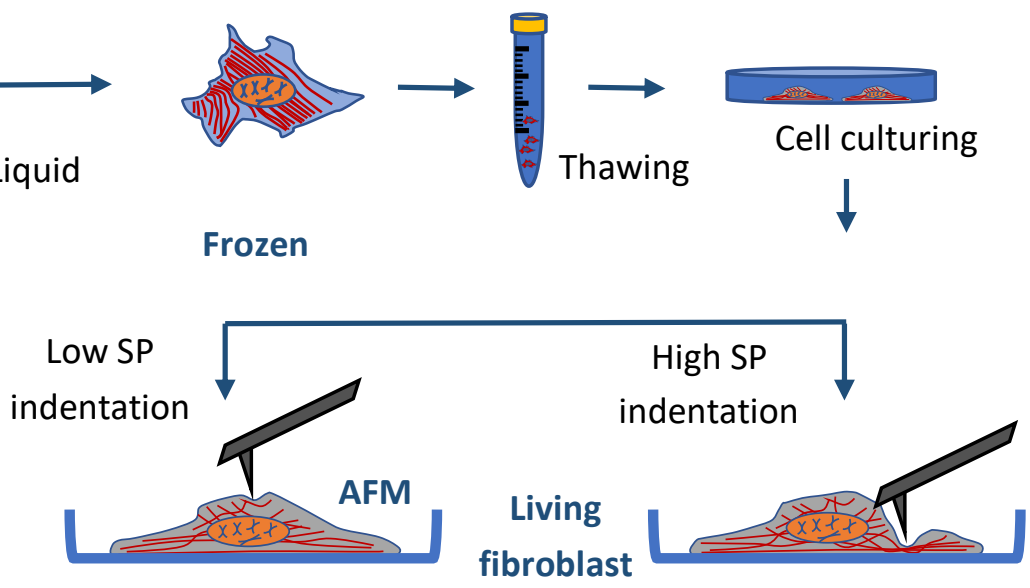

Figure 1 Overall schematic of the experiments. The mouse fibroblast cells are frozen in a liquid nitrogen after the standard incubation. Then the cells are thawed and the process of the cell development immediately after this is followed by the AFM nanoindentation method. High and low force

\section{EXPERIMENTAL PART}

\subsection{Freezing and thawing of the cells}

Fibroblasts from mouse embryos (mouse strain CF-1, MEFs) were cultured to 3rd passage (P3). Cell suspension was frozen, $10^{6}$ cells $/ 2 \mathrm{ml}$ cryo tube (TPP, Trasadingen, Switzerland). Drop by drop addition of $800 \mu \mathrm{l}$ of cold freezing medium into the same amount of cell suspension in MEF medium initiated the freezing process. MEF medium was composed of the following components: Knockout Dulbecco's modified Eagle's medium (KO-DMEM; Gibco), 10\% heat-inactivated fetal bovine serum (FBS; Invitrogen), 1\% non-essential amino acids (PAA), 1\% L-glutamine (Gibco), 1\% penicillin-streptomycin (PAA), and $0.1 \mathrm{mM} \beta$-mercaptoethanol (Sigma). Freezing medium was composed of KO-DMEM : FBS : DMSO in the following ratio: $3: 1: 1(\mathrm{v} / \mathrm{v} / \mathrm{v})$.

The cell thawing process was started, when the cryo tube with the frozen cells (MEFs P3) was taken out from liquid nitrogen. The tube was transported to the place of thawing on ice. Solid cell suspension was quickly thawed in the hot water until the moment, when a few last crystals could be visible in the tube. The whole content of the tube was then transferred to the standard Falcon tube, cold MEF medium (12 $\mathrm{ml}$ ) was drop wised by Pasteur pipette. After a short and gentle centrifugation of the tube ( $200 \mathrm{~g}, 4^{\circ} \mathrm{C}, 5$ minutes), the MEF medium excess was removed from the above of the cell pellet. Cells were then re-suspended in $1000 \mu$ of preheated MEF medium $\left(37^{\circ} \mathrm{C}\right)$. Thus, prepared suspension of fibroblast cells was seeded into the 3 plastic $34 \mathrm{~mm}$ plastic Petri dishes 93040 (TPP). Adhesion process was started in the standard $\mathrm{CO}_{2}$ incubator for about 20 minutes, then the dish was transferred to the JPK Petri Dish Heater (JPK, Berlin, Germany), where the nanoindentation process was started.

\subsection{Mechanical mapping of cells}

Silicon nitride cantilever Hydra 2R-100N (AppNano, Mountain View, CA, USA) with pyramidal silicon tip was used to characterize the cell mechanical properties. Cantilever stiffness and sensitivity was calibrated before each experiment, when the measurement setup was preheated to $37^{\circ} \mathrm{C}$ for at least half an hour (Petri Dish 
Heater, PDH, JPK). The laser reflection to the photodiode was optimized by the standard procedure described in the instrument manual. The AFM setup sensitivity was calculated as a slope of the force-distance curve (FDC) - length (Z-height) $450 \mathrm{~nm}$, curve was recorded at speed of $0.9 \mu \mathrm{m} / \mathrm{s}$. The sensitivity of the setup was typically in the range from 15.07 to $15.37 \mathrm{~nm} / \mathrm{V}$, stiffness of the cantilever was calculated by analysis of the thermal noise, and was found to be between 0.0173 and $0.0192 \mathrm{~N} / \mathrm{m}$.

JPK NanoWizard3 bioAFM microscope (JPK) was employed to characterize the cell mechanical properties in a real time following the thawing. AFM head for scanning by probe was placed on the inverted optical microscope Olympus IX-81. The optical microscope was used to find appropriate area containing a single fibroblast cell, which already started to adhere on a plastic bottom of the dish.

Mechanical properties of the cells were investigated by nano-indentation process, mode Force Mapping (JPK). Following experimental parameters were used: maximal loading force (Set Point) $1.0 \mathrm{nN}$ (relative to baseline value), curve recording speed $30 \mu \mathrm{m} / \mathrm{s}$, Z- length $15.0 \mu \mathrm{m}$. Force-distance curves were recorded with data sample rate of $2000 \mathrm{~Hz}$. Network of $64 \times 64$ points, so called Force Maps, were captured when area $55 \times 55$ to $75 \times 75 \mu \mathrm{m}$ was investigated.

Reproducibility of the nanomechanical measurement was performed by 5 times repeated force mapping process on identical place (scanning over the identical cell) with use of parameters mentioned above.

Place to place reproducibility was studied, when the identical force mapping experiments was subsequently performed on two different places found randomly on the Petri dish surface covered with fibroblasts. Effect of set point value on measured stiffness of the cells was studied, too.

Long term monitoring of cell mechanical properties during its development started 20 minutes after the cell thawing. When first cells started to adhere, culturing medium in the dish was completely exchanged and force mapping process was started. Nanomechanical measurement was performed for 12 hours of continuous experiment, when the Force Maps were recorded repeatably on the identical cell. The experimental parameters described above were employed, MEF medium preheated in the $\mathrm{CO} 2$ incubator was periodically exchanged every 60 minutes.

Networks of force distance curves (Force Maps) were processed by fitting the FDCs with Hertzian Sneddon equation (Eq. 1) thus providing value of Young's modulus. Data processing module of the JPK software was employed here, resulted Young's modulus maps were post-processed by Gwyddion software ver. 2.44X.

$F(\delta)=(2 E \tan \alpha) /\left(\pi\left(1-u^{2}\right)\right) \delta$

Where $F$ is the measured force, $E$-Young's modulus, $v$ - Poisson ration ( 0.5 for incompressible materials), $\delta$ is tip sample separation (obtained by correction of the cantilever height to its bending) and $\alpha$ is half angle to face of pyramidal tip (reflects the tip geometry).

Data post processing was initiated by threshold defined masking of the cell surrounding area presented by plastic material of high stiffness. The process of masking was finished by manual correction of mask by mask editor in Gwyddion software. Resulted Young's modulus maps contained only data points related to the area covered by cell. Average value of the cell stiffness was afterwards calculated by Statistical quantities module of the Gwyddion software.

\section{RESULTS AND DISCUSSION}

Mechanical properties of cell in the time period after the thawing were monitored. Storing cells in liquid nitrogen is a common method of storing them. The process of their thawing is relatively little studied, and the properties of the cells may be different from their normal behavior, which may We observed the mechanical properties of 
the cells in the time period after their thawing. The overall description of the freezing-thawing process combined with nanomechanical study is schematically described in the Figure 1.

The value of the maximum loading force (so called set-point) plays a critical role in the indentation process, higher value of the set-point leads to deeper indentation. Cell compartments located inside can be inspected by this way, on the other hand, sub-membrane structures can be selectively studied, when low forces are applied. Effect of the applied set-point value on measured values of the Young's modulus is shown in Figure 2. Measured cell stiffness increase with increased force value, however, only the lowest set-point leads to increased Young's modulus values, as only the membrane and close sub-membrane structure were investigated in this case.

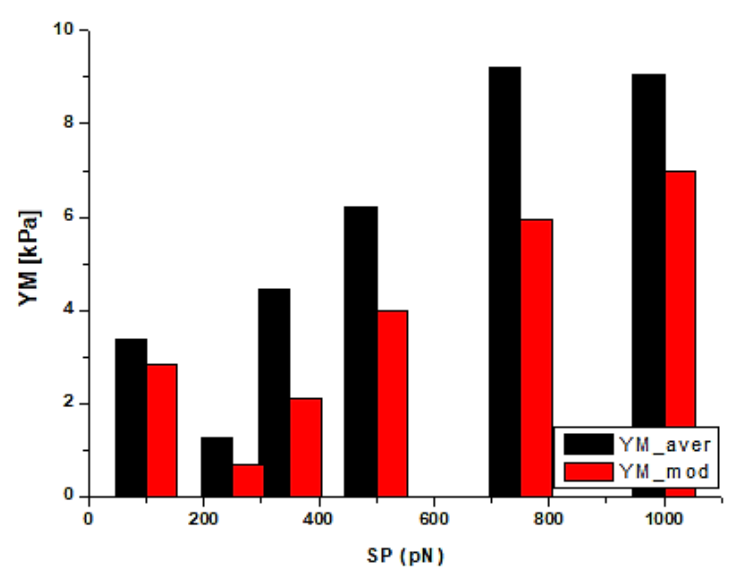

Figure 2 Effect of the loading force (set-point, SP) on the measured stiffness of the cell (YM, Young's modulus). Black columns show average values, red columns values modified values according to the substrate effect.
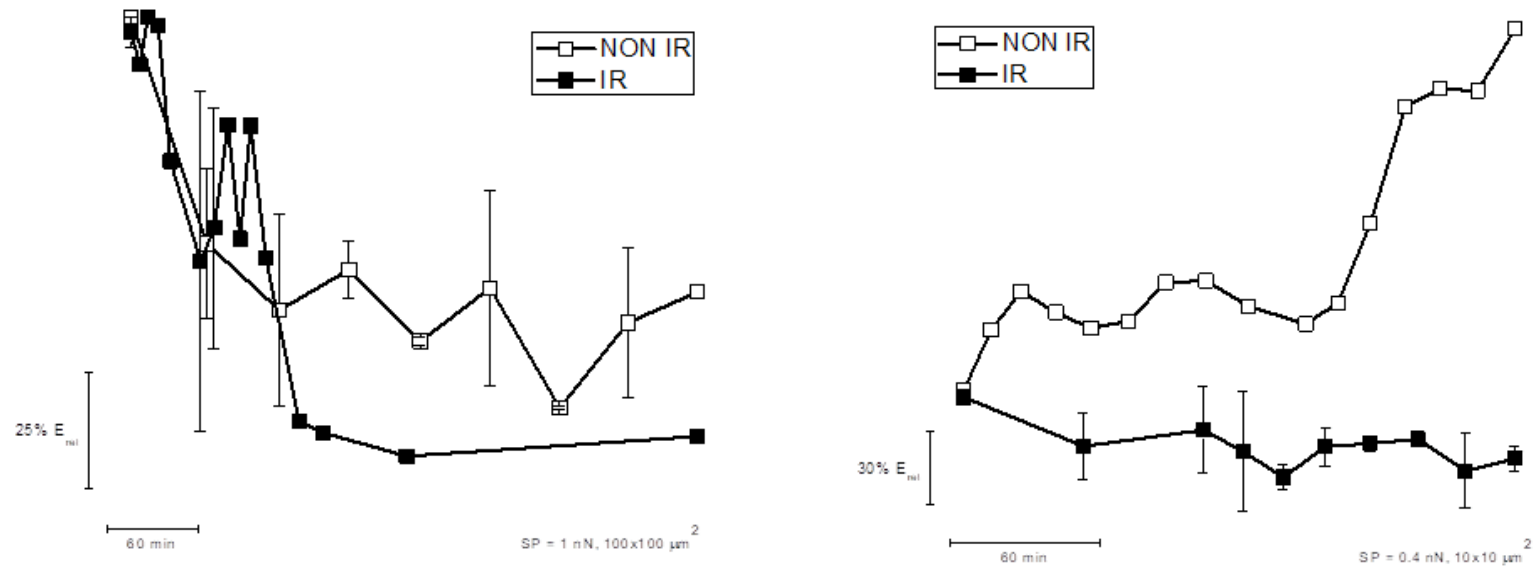

Figure 3 Time-lapsed progress of the average stiffness in the time, monitored in the time period immediately after the thawing process. Indentation set-point $1.0 \mathrm{nN}$ and $0.4 \mathrm{nN}$ was used for left and right graph, respectively. Area of $10 \times 10$ and $100 \times 100 \mu \mathrm{m}^{2}$ was monitored - graphs on the left- and right-hand side, respectively. Wild type (NON-IR) and mitotically inhibited (IR) by gamma irradiation were characterized in both cases.

Time lapsed monitoring of the Young's modulus of the freshly thawed cells is shown on the graphs (Figure 3). Deep indentation process with the set-point value of $1 \mathrm{nN}$ monitors the intracellular process. Cell stiffness decreases during the time period of 2 hours after the thawing, then the cell elasticity stabilize on stable level, 
which is about $25 \%$ higher in case of mitotically inhibited cells (gamma-irradiated), when this is compared with wild type non-treated cells.

Low force indentation (set-point value $400 \mathrm{pN}$ ) describes the cell development after the thawing procedure. Such a force leads to monitoring of membrane and sub-membrane structures. The indentation depth is only about $6 \%$ of the total cell height (approx. $550 \mathrm{~nm}$ ), comparing to the indentation depth of $2.5 \mu \mathrm{m}$ (approx. 30\% of the total cell height), when the higher set-point is used. Totally different view of the cell status can be seen when the low indentation method is used. Sub-membrane structures of the WT cells are rapidly stiffening for the whole time period of the monitoring, as the cells can normally develop. On the other hand, the mitotically inhibited fibroblast cells cannot process the regular cell cycle, thus keeping the stiffness of the sub-membrane structures on a constant level.

\section{CONCLUSION}

Mechanic and elastic properties of a cell are closely related to its function. Some aspects of cell's internal working and interactions with its surroundings are based upon mechanical as well as biochemical principles. In this work, we showed the ability of the AFM-based nano-indentation method to study the overall cell mechanical properties in a real time. The biological example of the cell regeneration after the thawing was chosen to illustrate the method ability to monitor cell membrane, sub-membrane and internal content changes. Low and high force indentation was applied to study sub-membrane and intracellular cellular structures. Moreover, time-lapsed monitoring of the cellular stiffness has led to an interesting view of the cell development during the post thawing period. Presented work offers an interesting methodological approach for various biological experiments dealing with changes of the cell stiffness.

\section{ACKNOWLEDGEMENTS}

\section{Supported by Ministry of Health of the Czech Republic, grant AZV NU20-06-00156, we acknowledge CF Nanobiotechnology supported by MEYS CR (LM2018127).}

Supported by the project no. LQ1605 from the National Program of Sustainability II (MEYS CR) project FNUSA-ICRC and Supported by the European Regional Development Fund - Project ENOCH (No. CZ.02.1.01/0.0/0.0/16_019/0000868). Martin Pes/ was supported by "Junior researcher 2020" scheme, Medical faculty, Masaryk University.

\section{REFERENCES}

[1] NARDONE, G., CRUZ, J.O.-D.L., VRBSKY, J., et al. YAP regulates cell mechanics by controlling focal adhesion assembly. Nature Communications. 2017, vol. 8, article no. 15321.

[2] GOLAN, M., JELINKOVA, S., KRATOCHVÍLOVÁ, I., et al. AFM Monitoring the Influence of Selected Cryoprotectants on Regeneration of Cryopreserved Cells Mechanical Properties. Frontiers in Physiology. 2018, vol. 9 , p. 804.

[3] GOLAN, M., PŘIBYL, J., PESL, M., et al. Cryopreserved Cells Regeneration Monitored by Atomic Force Microscopy and Correlated With State of Cytoskeleton and Nuclear Membrane. IEEE Transactions on NanoBioscience. 2018, vol. 17, no. 4, pp. 485-497.

[4] RAUDENSKA, M., KRATOCHVILOVA, M., VICAR, T., et al. Cisplatin enhances cell stiffness and decreases invasiveness rate in prostate cancer cells by actin accumulation. Scientific Reports. 2019, vol. 9, no. 1, pp. 1-11.

[5] PERESTRELO, A.R., SILVA, A.C., OLIVER-DE LA CRUZ, J., et al. Multiscale Analysis of Extracellular Matrix Remodeling in the Failing Heart. Circulation Research. 2020.

[6] CALUORI, G., PRIBYL, J., PESL, M., et al. Advanced and Rationalized Atomic Force Microscopy Analysis Unveils Specific Properties of Controlled Cell Mechanics. Frontiers in Physiology. 2018, vol. 9, p. 1121. 
[7] DENG, X., XIONG, F., LI, X., et al. Application of atomic force microscopy in cancer research. Journal of Nanobiotechnology. 2018, vol. 16, article no. 102.

[8] PARK, S., LEE, Y.J. AFM-based dual nano-mechanical phenotypes for cancer metastasis. Journal of Biological Physics. 2014, vol. 40, no. 4, pp. 413-419.

[9] ELSAYAD, K., POLAKOVA, S., GREGAN, J. Probing Mechanical Properties in Biology Using Brillouin Microscopy. Trends in Cell Biology. 2019, vol. 29, no. 8, pp. 608-611.

[10] GONZÁleZ-BERMúdEZ, B., GUINEA, G.V., PLAZA, G.R. Advances in Micropipette Aspiration: Applications in Cell Biomechanics, Models, and Extended Studies. Biophysical Journal. 2019, vol. 116, no. 4, pp. 587-594.

[11] WU, P.H., AROUSH, D.R., ASNACIOS, A., et al. A comparison of methods to assess cell mechanical properties. Nature Methods. 2018, vol. 15, no. 7, pp. 491-498. 